\title{
The Emergence of Resourced Research Services
}

Sandra Lawson $^{1^{*}}$, Susan Seroskie ${ }^{1}$, Tess Mayall ${ }^{2}$, Brian Douglas ${ }^{3}$ and Les Jebson ${ }^{4}$

${ }^{1} R N$, Executive Vice President, Strategic Resourcing, Advanced Clinical, USA

${ }^{2}$ Community Manager, Science Exchange, USA

${ }^{3}$ CFA, CFO, ProofPilot, USA

${ }^{4}$ Administrator and Adjunct Lecturer, University of Florida Diabetes Institute, USA

"Corresponding author: Sandra Lawson, RN, Executive Vice President, Strategic Resourcing, Advanced Clinical, USA, Tel: 352-281-3911; E-mail: lawsones@ufl.edu

Rec date: Nov 12, 2014, Acc date: Apr 01, 2015, Pub date: Apr 05, 2015

Copyright: ( 2015 Lawson S, et al. This is an open-access article distributed under the terms of the Creative Commons Attribution License, which permits unrestricted use, distribution, and reproduction in any medium, provided the original author and source are credited.

\section{Introduction}

An integral component of many institutions of higher learning is that of a robust scientific mission. Historically, universities and research foundations have attempted to recruit established scientists from other institutions or attempted to incubate and develop new post doctorate researchers or research teams, often referenced as junior faculty' or 'promising faculty'. These efforts are often with the intent of making scientific advancements in basic and translational research, and to strengthen the overall funded research portfolio and rankings of the respective university. However, more often than not, organized scientific research can be a financial and overall resource intensive undertaking. Millions of personnel hours and hundreds of millions of dollars are spent annually, in providing and supporting organized 'start-up' packages from the sponsoring institution, colleges and departments for researchers. Unfortunately, it is not unusual for some of these programs to sputter or not achieve the financial or scientific returns that may have been anticipated. It is not unusual for a 'start up' or 'recruitment' package for an individual researcher or their team to be anywhere from $\$ 500,000$ to $\$ 1,000,000$ or more annually over the life of the agreement. Ironically, the science behind incubating and developing such programs in these settings has historically, been less than scientific in means and management. These 'start up' monies are often outlined in the new program or researcher start up agreement, and the funding is intended to allow the new faculty and team members to purchase the basic instrumentation and equipment as well as support employees in the form of grant writers, assistants and postdoctoral researchers. This funding is finite, with the understood expectation that the faculty member and research program will seek and obtain funding from an outside agency, such as the NIH or other foundational funding, and thus reduce or ultimately eliminate the need for sponsoring institutional funding. It often includes the provision for administrative office and lab space, for which the costs of construction or maintenance are not included, but are frequently a sizable component. The National Institutes of Health (NIH) and the National Science Foundation (NSF) are the two major government funded bodies that provide research funding to scientists, with the NIH spearheading grant allocation to research that has health relevance and the NSF funding basic science. In the most recent funding numbers, the NIH was allotted $\$ 31.2$ billion dollars while the NSF was allotted $\$ 6.5$ billion. According to the $\mathrm{NIH}$, one in five grant applications secure funding. The lag period from grant application, to approval of funds being released can reach almost a year in duration.

Needless to say, raising the prestige of a university, advancing the field of science and the peripheral economic implications are all worthy and prudent reasons for pursuit and development of promising and eminent researchers and associated programs.

However, we believe it is prudent to give consideration to the emergence of resourced components of new program establishment as an important component. Specifically, with the goal of more effectively managing financial risk, and reducing the time span for new research implementation; that resourcing specific functionality can be an important asset.

The concept of resourcing specific facets of organized research has roots in the private biotechnology research space. It is not until more recently that universities and research foundations have begun to explore resourcing as an important financial component of program development or sustainability.

The resourcing value proposition lies in the fact that the same desired aforementioned objectives can be met, but with the following additional benefits:

- A markedly faster start up window for getting specific research underway.

- Elimination or reduction in the construction, maintenance and utilization of research facility space.

- Reduction of or elimination of redundant lab equipment and personnel.

- The ability to manage the financial risk of a new program with far less capital outlay.

- The ability to support and activate multiple new researcher and program start-ups simultaneously.

With the intent of vetting the feasibility of creating a virtual research laboratory, it is prudent to break down and examine the potential benefits and drawbacks behind four critical core areas, which include: personnel, information technology, scientific equipment and experiments, and facility space.

\section{Personnel-staffing models}

When considering how to resource key personnel for a new program development, there are several types of models that can be implemented in order to provide the right resources, and each model comes with its own set of benefits and risks. Determining the right model begins with an assessment of the project's needs and goals. Do you need personnel on location? Do you need personnel working across multiple projects or just one project? Do you want to manage the personnel or do you want an outside provider to manage them? Do you need one person or a team? 


\section{Insourced-traditional staffing}

Implementing an insourced model means hiring permanent, fulltime and/or part-time employees into a company and providing them with benefits, performance management, and career development.

Benefits: Hiring directly offers immediate flexibility to move an employee from one project to another. This provides a continuity of dedicated resources at any time. You will often pay a lower hourly rate than if you were to outsource personnel through an agency. Lastly, employees are trained to the company's standard operating procedures (SOPs) and therefore provide quality deliverables.

Risks: Generally speaking, when work volume is low, employee fixed salary costs can be high. However, when high work volume warrants a ramp up in resources, scaling to accommodate the work volume may be difficult trying to find and hire the right resources in an efficient manner. It often can take several months to recruit and train research support staff. Another challenge to an insourced model is employee turnover and performance management. If personnel should leave in mid-project, or are performing at a below-average standard, this could negatively affect the project causing delays in timelines and thus raising budgets. It is also important to note that insourcing models have overhead and line management responsibilities to manage the employees, in addition to IT infrastructure costs.

In a traditional staffing model, a university or host institution is hiring personnel through an external provider on an as-needed basis to fulfill a role in the organization. Wages are typically paid at an hourly rate, benefits and PTO may vary, and there is no dedicated oversight of the personnel by the provider. Key positions such as grant writer or clinical coordinator can be hired and start within days instead of months.

Benefits: Simply put, this is the most flexible resourcing model and provides an interim strategy while long-term plans are put into place.

Risks: The most common risk in this type of model is having an internal infrastructure in place at the company to manage and train new staff. In addition, there is a loss of efficiencies as contracts end and personnel leave the organization-the cycle of onboarding and training starts over with new personnel.

\section{Functional service model}

Functional models provide the most structure in terms of resourcing. In this type of model, personnel are contracted through an external provider for one or more functions. Functional models can work across projects or programs, offer co-employment risk mitigation, provide overall model management and oversight, and use fixed-fee type pricing. Additionally, personnel benefits and PTO are consistent among hires because they are established through the external provider.

Benefits: Resourcing using a functional model provides flexibility in that personnel can be dedicated to individual projects or an entire program. Contracting functional area subject matter experts for a project or program creates succinct, best-in-class processes thus creating efficiencies. Another benefit of this type of model is reduced training costs. When functional resources are hired to work across multiple projects or programs, it eliminates the need to re-train new resources for new projects. Finally, functional models offer key performance indicators (KPIs) and metric tracking, as well as quality control in that the ownership of projects stays with the client.
Risks: There are several considerations to be mindful of when resourcing as a functional model. The first is that success within this type of model is greatly dependent on the client's infrastructure and whether or not the company culture is a good fit. There could also be a concern for career development in a long-term commitment which may affect attrition within this model. And while efficiencies are created by providing succinct processes, these efficiencies may not be recognized immediately.

\section{Information technology and data management}

The concepts of just-in-time, cloud computing, software-as-aservice, and outsourced services have dramatically improved business processes and results in many major industries. As these concepts take root in academia they have the potential to create substantial opportunities well beyond decreased cost and increased efficiency. As hundreds of cloud-based resources have sprung into existence it can be overwhelming for scientists to navigate and select from the diverse offerings available.

Data is paramount to nearly every scientist's research. Data files as big as 100 GB are now regularly generated in biomedical research. In the past, the primary modality in which to share information was through conventional mail. Because of file size, conventional email sharing has not been viewed as a secure or viable model.

However, today there are many services that are free of charge or are a small cost that address the challenge of large scientific data storage and sharing. An example of this is 'Figshare' which is a data repository and sharing site where researchers can upload and store their data for free, as long as it is publicly available, or for a premium they can store their data privately. 'Globus' is another data sharing platform that was created specifically for sharing the massive amounts of data that are created in biomedical research, and because they don't use external storage, files of any size can be shared between researchers. Both of these platforms were specifically created to make it easy for scientists to store and share data.

Using the many software-as-a-service marketplaces scientists can easily outsource website generation and hosting services should they not be available from the host institution. For website creation, contractor marketplaces such as eLance and oDesk are ideal, because they allow you to browse and select website designer professionals. Websites can be hosted using Hostgator or BlueHost. In total, researchers can build and host their websites for as little as $\$ 1000$ $\$ 2000$ and maintain total control of the end result in the process.

In addition, results from the research administration software industry indicate that the cost of using commercially available software platforms like ProofPilot, a tool to design and automate human subjects research, may be less than $50 \%$ of that of traditional methods. Such platforms significantly reduce research study set-up time (by about two thirds), require fewer staff hours as many research activities are automated and can be turned on and off as needed, and reduce many costs - such as website hosting and database management associated with studies. That said, software-as-a-service goes beyond web development and data storage to augment investments in human resources and improve operational efficiency by automating and managing complex research operations. The private sector has realized massive efficiencies with specialized systems for accounting, logistics, human resources and many other back office operations. Increasingly, venture-backed companies like ProofPilot are creating solutions specifically for academic researchers. They provide an extensive set of 
features - many beyond the scope of even the largest human subjects research lab - at price points that are within the scope of a small research study budget.

\section{Laboratory facility and equipment considerations}

Sometimes equipment that has been purchased prior is available at no cost for new research lab start-ups. However, more often than not, our experiences have been one of not completing a thorough inventory evaluation beforehand to validate available equipment resources, but rather to provide funding for the new research program to purchase new equipment specific to their own program. In addition, the suggestion that scheduling and logistical challenges surrounding sharing of research equipment can be prohibitive. Considerable variation exists in the capital costs associated with new health sciences research implementation, and for the purposes of our discussion, we submit that these expenses could be eliminated if it was validated that the associated specific experiments could instead be procured through scientific exchanges or fee-for-service laboratories. It can also be considerably taxing for research programs when specific equipment malfunctions or requires repairs; which locating a dedicated service employee for the equipment can be a challenge. Annual service contracts that cover maintenance responsibilities for such equipment can range from $\$ 5,000$ on an analytical ultracentrifuge to $\$ 100,000$ or more on a state of the art transmission cryo- electron microscope.

Furthermore, the cost of lab "consumables" can be anywhere from $\$ 20,000-\$ 50,000$ or more per fiscal year, with the cost increasing with an increase in the number of people in a lab and the price of special reactants.

However, with thousands of fee-for-service laboratories in existence, it can be, in itself, time prohibitive to choose the correct lab for a researcher's analysis. Science-as-a-service marketplaces were born as a solution to this problem. One such example is California based, Science Exchange. Science Exchange is a marketplace for scientific experiments where scientists can browse, compare, and order experiments from labs around the world. It can be likened to a sort of 'priceline.com' model where laboratories around the country offer to provide experiments at various pricing models. At present, Science Exchange has over 900 labs providing over 2000 different experiment types.

'Benchspace' rentals is a model that offers an ideal alternative to traditional labs. Programs such as QB3 and Harlem Biospace rent space where researchers can do sample prep and preliminary research on a monthly basis. Additionally, reagents and scientific products can be bought cheaply through online vendors such as HappiLabs and P212121. Even a scientist's lab management can be outsourced utilizing free software services such as Quartzy.

Taking lessons from the software-as-a-service development, scienceas-a-service is a recent development that enables virtual laboratory work. For years scientific specialization and collaboration have been on the rise, but only recently have efficient, market-driven collaboration options emerged.

Benefits: By monetizing laboratory experiments, core facilities and contract research organizations give researchers the option to complete experiments without investing in costly infrastructure. Additionally, based on our estimates, researchers could save between $80-90 \%$ by outsourcing common experiment types such as molecular cloning or RNA extraction. Lastly, instead of learning each new technique necessary for research, scientists can save massive amounts of time by outsourcing to experts. As a result, the fee-for-service model has the potential to speed up the scientific process and decrease its cost.

Risks: Because the fee-for-service model is in its infancy, there are several risks to consider before implementing this process. Most importantly, it can be difficult for scientists to feel comfortable with an unknown lab handling their samples. This can be solved with thorough phone calls and e-mails, which can be time consuming. Shipping samples can be costly, and must be factored into a scientist's decision to outsource their work. As time passes, many of these risks will decrease, but it will take time to perfect the system.

\section{Concluding Thoughts}

The case for resourcing specific components of organized research has measurable merit and benefits, whether it is in the form of partial resourcing of personnel, or deferring the capital outlay in purchasing and operating expensive equipment. While the costs associated with the construction of large bays for bench research are often in the millions of dollars to construct and maintain, conventional institutional models still prevail at many universities around the country. However, when we compare and integrate all of the prudent considerations of time, people and space - we believe that the creation of hybrid resourced laboratory programs will not only prove to be markedly more financially effective, but will also allow for more timely advancements in the field of basic and translational health sciences research. 